\title{
EDITORIAL
}

\section{COVID19 VACCINATION IN FRAIL PEOPLE. LOTS OF HOPE AND SOME QUESTIONS}

\author{
Y. ROLLAND ${ }^{1}$, M. CESARI ${ }^{2}$, J.E. MORLEY ${ }^{3}$, R. MERCHANT ${ }^{4}$, B. VELLAS $^{5}$
}

1. Service de Medecine Interne et Gerontologie, Gerontopole, Toulouse, France; 2. Geriatric Unit, IRCCS Istituti Clinici Scientifici, University of Milan, Milan, Italy; 3. Division of Geriatric Medicine, Saint Louis University School of Medicine, St. Louis, Missouri, USA; 4. Division of Geriatric Medicine, Department of Medicine, National University Hospital, Singapore, Singapore and Department of Medicine, Yong Loo Lin School of Medicine, Singapore, Singapore; 5. Gérontopôle UMR Inserm 1027, Université Paul Sabatier, CHU

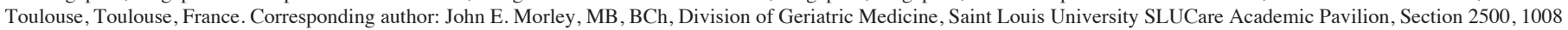
S. Spring Ave., 2nd Floor, St. Louis, MO 63110, USA, Email: john.morley@health.slu.edu, Twitter: @drjohnmorley

Key words: COVID19 vaccination, frail, elderly.

"Vaccines are the tugboats of preventive health" William Foege

In 2020, the world was devastated by the COVID19 pandemic $(1,2)$. At this stage it is clear that the persons most likely to die from COVID19 are in the elderly population, especially those in Nursing Homes. Nursing home residents represent $0.6 \%$ of the population in the USA, is $0.7 \%$ of the Italian population, and $1.1 \%$ of the French population. However, nursing home residents account for 30 to $40 \%$ of all deaths from COVID-19. Given the estimated 100,000 deaths in the US, 14,000 in Italy, and 18,000 in France caused by the COVID-19 among residents of long-term care facilities, it is unfortunate that the study by Polack et a (3) did not involve the population most affected by the disease. The oldest participant was 83 , but very few were older than 75 . Exclusion for research of frail older people is common, but can be considered an unacceptable example of ageism.

The first phase of vaccination in many countries is focused on nursing home residents. Residents of long-term care facilities will be the first population to experience, in real life, the benefits and potential sides-effects of the new vaccines. There are multiple new vaccines that have been developed (Table 1) (4). Frail older adults are receiving the vaccine, but were not included in the trials. Unexpected safety issues may arise when millions of very frail older adults such as nursing home resident will be vaccinated around the world. Given the usual rate of clinical events among frail older adults, recording side events and defining accountability of the vaccine will be challenging. The rate of death is around 30\% per year in nursing homes, and questions will arise if a death occurs in the period following vaccination. Further there is a high expected incidence of side effects when the vaccine is given to persons who recently had COVID19 infection.

Human aging is characterized by physical and physiological changes that reduce the immune system and modulate vaccine responses $(5,6)$. Whether the coming COVID vaccine will generate an effective response in the oldest and frail older people and how these responses will be modulated by age or other clinical factors such as frailty is currently an open question. In older individuals who show increased COVID19 disease severity, an impairment in the protective effect of COVID-19 vaccine responses in older adult may be observed. Preliminary data suggests a reduced antibody response to COVID19 vaccine in older persons.

Table 1

Vaccines for COVID19
1. Moderna (mRNA)
2. Pfizer/BioNTech (mRNA)
3. Oxford/AztraZenecaAZD1222 (Modified Adenovirus)
4. Sinovac; China (Inactivated live virus)
6. SputnikV; Russia (Modified Adenovirus)
7. Covidicea; China (Inactivated live virus)
8. Soberana; Cuba (spike protein antigen)
9. Novovax (protein adjuvant)
10. Jansen Ad28CoV2.5 (Modified adenovirus)
11. GSK/Sanofi (protein adjuvant)
12. Valneva (Inactivated live virus)

Lockdown during the COVID19 pandemic has led to older persons living at home and especially those in nursing homes to experience increased isolation and loneliness $(7,8)$. Loneliness leads to depression, poor sleep, decreased cognition, cardiovascular disease, disability and death (9). Staff wearing Personal Protective Equipment further causes socialization distance. This has created a major reason for older persons and their carers to receive the COVID19 vaccination.

Despite the tremendous success of vaccines at decreasing illness and mortality there is a high rate of vaccine mistrust around the world. This is fueled by "false facts" being disseminated by anti-Vaxers through social and at times mainstream media. In some parts of the world, such as France, Ukraine, Russia and Japan, under $50 \%$ of the population do not believe vaccines are safe. (https://www.weforum.org/ Published online January 20, 2021, http://10.1007/s12603-021-1591-9 
agenda/2019/09/this-is-how-attitudes-to-vaccines-comparearound-the-world/). In the USA, surveys have found that between 50 to $70 \%$ of persons are willing to have the COVID19 vaccine.

Another problem is obtaining consent for the vaccine in older vulnerable persons. This includes the decision of whether cognitively impaired persons can give their own consent or need consent from a designated relative. This may limit access to vaccination for the most vulnerable who are not able to give their consent. In some countries the request for consent is seen by patients and proxy as a disengagement of family physicians and may generate mistrust of the vaccine.

The availability of vaccines for COVID19 represents the clearest way to reverse the COVID19 pandemic. It is clear that older persons are at the greatest risk of disease and poor outcomes from it. This includes not only acute illness and mortality but also the symptoms associated with long COVID (10). Questions remain about its efficacy in frail older persons, its potential side effects and how often the population will need to be revaccinated. Finally, it will be necessary to determine which is the most cost-effective vaccine.

Conflict of interest: The authors declare there are no conflicts of interest

\section{References}

1. Morley JE. Editorial: 2020: The Year of The COVID-19 Pandemic.J Nutr Health Aging. 2021;25(1):1-4

2. Morley JE, Vellas BJ. Editorial: COVID-19 and Older Adults. J Nutr Health Aging. 2020;24(4):364-365

3. Polack FP, Thomas SJ, Kitchin N, et al. Safety and Efficacy of the BNT162b2 mRNA Covid-19 Vaccine. N Engl J Med. 2020.

4. Soiza RL, Scicluna C, Thomson EC. Efficacy and safety of COVID-19 vaccines in older people. Age Ageing. 2020

5. Sewell HF, Agius RM, Kendrick D, Stewart M. Covid-19 vaccines: delivering protective immunity. BMJ. 2020

6. Takeda C, Angioni D, Stephan E, et al. Age-Related Frailty: A Clinical Model for Geroscience? J Nutr Health Aging. 2020;24(10):1140-1143

7. Astrone P, Cesari M. Integrated Care and Geriatrics: A Call to Renovation from the COVID-19 Pandemic. J Frailty Aging. Published online October 28, 2020:1-2. doi:10.14283/jfa.2020.59

8. Sepulveda-Loyola W, Rodriguez-Sanchez I, Perez-Rodriguez P, et al. Impact of Social Isolation Due to COVID-19 on Health in Older People: Mental and Physical Effects and Recommendations. J Nutr Health Aging. 2020;24(9):938-947

9. Berg-Weger M, Morley J. Editorial: Loneliness in Old Age: An unaddressed Health Problem. J Nutr Health Aging. 2020;24(3):243-245.

10. Morley JE. Editorial: COVID-19 - The Long Road to Recovery. J Nutr Health Aging. 2020;24(9):917-919. 